\title{
Application of TOPSIS and AHP in the Multi-Objective Decision-Making Problems
}

\author{
Yihan Wang ${ }^{1, a}$ \\ ${ }^{1}$ School of Management, Shanghai University, Shanghai, China
}

\begin{abstract}
The problems of multi-objective decision making are analysed and studied. In order to solve its optimization problems, the basic principles and application steps of TOPSIS and AHP are introduced in this paper. Then some practical examples are given to show how to apply these two new methods in multi-objective decision making problems. Finally, the advantage and feasibility of the TOPSIS and AHP methods are demonstrated by theoretical analysis.
\end{abstract}

\section{Introduction}

TOPSIS and AHP, both qualitative and quantitative analysis methods, have strong applicability and effectiveness in dealing with complex multi-objective decision-making problems in lives and at works. They can be applied into a wide range of fields, such as, the whole economic plan and administration, the proper allocation and utilization of energy, social and behavioural sciences, military multi-target decisions, transport and finance problems, agricultural and environmental development, education and talents, health and social securities.

Accelerating production and circulation of data is a fact of today's life for the rapid development of cloud computing, information technology and big data era. Therefore, it is of great importance to effectively extract conductive and valuable information when we encounter massive data to process, and this large amount of data is still increasing in a geometrical rate. Mastering these two methods can help us not only to form a mechanism concerning how to conduct a statistical analysis of the mass data, let alone deep analysis and apply the mechanism at certain scale, but also to use and select important information in mass data and make better decisions.

\section{Multi-objective decisions making}

\subsection{Definition}

Multi-objective decisions making is a process of decision making with more than two decision-making goals. The multiple criteria and rules are needed when evaluating and optimizing the decision-making problems. For instance, we consider a variety of factors -- fashionable style, excellent quality and low price -- when buying clothes. This is a simple but common multi-objective decision- making case in our daily life. Living in an era of technological advances and information explosion, it is very important to make a good decision based on systematic and scientific methods.

\subsection{Main characteristics}

The target goal of the multi-objective decision-making problems has two main characteristics, that are, incommensurability and contradiction.

On one side, incommensurability refers to the fact that there is no uniform standard or measurement unit for each target.

On the other side, contradiction means that there often exists paradox and conflicts among different goals. For example, products with lower prices are often of lower quality than those with higher prices, so we are not likely to pick a low-cost but good-quality goods.

As a result of non-commensurability and contradiction on multi-objective decision, decision behaviour is more complicate. The value orientation and multi-objectives often are not consistent. Frequently, there are paradoxes and non-uniform measurement in decision process.

\subsection{Classification}

The multi-objective decision-making problems can be customarily divided into two categories based on the number of alternatives. One is the multi-attribute decisionmaking problem with limited number of schemes. The other is multi-objective decision-making problem with infinitely multi-schemes.

The similarities between these two kinds of decisionmaking problems lies in that they must make decisions on the quality, the merits and demerits of each scheme with more than one criteria. In most cases, these criteria and rules are not fair or even contradictory.

\footnotetext{
a Corresponding author: 399909527@qq.com
} 
The difference among them are: (1) The decision space of the former is discrete, but the latter is continuous. (2) The solution to the former is limited and known, while the latter is infinite and unknown. (3) The constraints of the former are implicit in the criteria and do not play a direct role, whereas, the latter are beyond the criteria and play an indispensable part. (4) The core of solving the former problem is to evaluate and schedule each alternative, then choosing the best scheme by weighting their pros and cons, however, the efficient solution to dealing with the latter problem is utilizing the mathematical vector optimization.

\subsection{The constituent elements}

\subsubsection{Decision unit}

The decision-making unit is the principle part of the decision-making process, including accepting assignments, inputting information, generating internal information, forming system knowledge, providing valuable judgments, and thus achieving the final decision. The decision-making unit is composed of decision makers, decision analysts and information processing equipment.

\subsubsection{Target set}

Establishing a relatively stable target set for multiobjective decision-making problems. To clarify the goal, it can be expressed as a hierarchy. The top layer, indicating people's general expectations and requirements for the problem, is a general description and not easy to operate directly. So, the overall goal needs to be broken down into several alphabet levels. In addition, the tree structure is usually used to visually represent the hierarchy of the target criteria system.

\subsubsection{Attribute sets}

After determining the target set of multi-objective decision, a certain method and means are needed to measure the bottom target layer. The attribute sets must content two properties: comprehensibility and testability. That is to say, the attributes should fully reflect the degree of reaching goal on multiple assignment operations in a given plan.

A complete attribute set should generally meet the following requirements: (1) Completeness. In order to make a correct decision, the set of attributes should reflect all the important aspects of the decision problem. (2) Nonredundancy and simplicity. Preventing a target from being reconsidered and affecting the decision results. Moreover, attribute sets should be computable, decomposable and minimal for effective statistics and analysis.

\subsubsection{Decision-making situation}

Decision making situation refers to the reality and dynamics of decision-making. It includes two aspects, namely, decision-making environment and decisionmaking structure.

Decisions making depends on the exterior environment.
If there were no concrete contexts and occasions, the decision will lose time, space and conditions. The possibility and reality of decision-making, as a result, are totally lost. Therefore, a correct comprehension of the decision-making environment is the prerequisite for making correct decisions.

Decision-making structure refers to all kinds of essential elements and internal relations that run through the whole decision-making process. It is the basis for the occurrence of decision-making behaviour, and the common or regular embodiment nature of decisionmaking activities.

\subsubsection{Decision-making rules}

Decision-making rules refer to the procedures used for sorting and comparing scenarios. The rules must be coordinated with decision-making goals. There are two categories of the decision-making rules. One is the optimal rule that fully serializes the plan. And the other is a satisfactory rule, which sacrifices the optimality and simplify of problems.

\section{Multi-objective decisions making based on TOPSIS}

\subsection{Basic principles of TOPSIS}

In multi-objective decision-making problems, we always intend to find a solution that performs best among all attributes. This is what so-called the best solution. Such a scheme, however, generally does not exist in many practical problems, and the performance of alternatives have their own strengths and weaknesses respectively in the different subsets.

The TOPSIS (Technique for Order Preference by Similarity to an Ideal Solution) method, evaluating the relative pros and cons of existing objects, is based on the close degree of limited evaluation objects and idealized targets. It takes "positive-ideal solution" and "negativeideal solution" as reference datum. Meanwhile, the Euclidean Distance is adapted to measure the distance of every concept to the positive-ideal solution and the negative-ideal solution

For one thing, the positive-ideal solution, whose various attribute values reach the best in each alternative, is the best solution or scheme among all alternatives. It performs best but often not exist. In particular, the positive-ideal solution is considered as a reference datum point. The closer to the positive-ideal solution point, the better the scheme is. For another thing, the negative-ideal solution is regarded as another reference datum point, which is the worst scheme or project, and its various attribute values reach the weakest among all alternatives.

In the end, what we supposed to do is to rank the advantages and disadvantages of each alternative. Comparing each alternative with the ideal solution and the negative ideal solution enables to provide objective basis for evaluating all schemes. The best scheme is the closest to the positive-ideal solution, at the same time, the farthest 
from the negative-ideal solution.

\subsection{Practical application of TOPSIS}

There are many applications of the TOPSIS method in daily life. For example, the decision maker X1 who intends to go to $4 \mathrm{~S}$ shop to buy a car, makes a comprehensive evaluation of different types of cars (A1-A4) according to five attributes -- Maximum speed (C1), Price (C2), Acceleration time (C3), Reliability (C4), Sensitivity (C5). Table 1. shows the specific attribute values of A1-A4 cars.

Table 1. Selection information list of 4 types of cars

\begin{tabular}{|c|c|c|c|c|}
\hline & A1 & A2 & A3 & A4 \\
\hline C1 & $150 \mathrm{~km} / \mathrm{h}$ & $200 \mathrm{~km} / \mathrm{h}$ & $180 \mathrm{~km} / \mathrm{h}$ & $160 \mathrm{~km} / \mathrm{h}$ \\
\hline C2 & $¥ 100,000$ & $¥ 400,000$ & $¥ 200,000$ & $¥ 150,000$ \\
\hline C3 & $10 \mathrm{~s} / 100 \mathrm{~m}$ & $12 \mathrm{~s} / 100 \mathrm{~m}$ & $14 \mathrm{~s} / 100 \mathrm{~m}$ & $15 \mathrm{~s} / 100 \mathrm{~m}$ \\
\hline C4 & Low & Fair & High & Fair \\
\hline C5 & Fair & Fair & High & Fair \\
\hline
\end{tabular}

\subsubsection{Constructing weighting-standardized matrix}

Standardizing decision matrix by vector normalization method:

$$
Y=\left[\begin{array}{lllll}
0.4321 & 0.2074 & 0.3878 & 0.4811 & 0.6708 \\
0.5762 & 0.8296 & 0.4653 & 0.2887 & 0.3127 \\
0.5185 & 0.4148 & 0.5429 & 0.6737 & 0.5217 \\
0.4609 & 0.3110 & 0.5817 & 0.4811 & 0.3727
\end{array}\right]
$$

Data sheet of pair-wise comparison:

Table 2. Data sheet of pair-wise comparison

\begin{tabular}{|c|c|c|c|c|c|}
\hline & C1 & C2 & C3 & C4 & C5 \\
\hline C1 & 0.5 & 0 & 0 & 0.5 & 1 \\
\hline C2 & 1 & 0.5 & 0 & 0.5 & 1 \\
\hline C3 & 1 & 1 & 0.5 & 0 & 1 \\
\hline C4 & 0.5 & 0.5 & 1 & 0.5 & 0 \\
\hline C5 & 0 & 0 & 0 & 1 & 0.5 \\
\hline Sum & 3 & 2 & 1.5 & 2.5 & 3.5 \\
\hline Weight & 0.24 & 0.16 & 0.12 & 0.2 & 0.28 \\
\hline
\end{tabular}

The weight value is calculated by pair-wise comparison data of table 2 :

$\mathrm{W}=(0.24,0.16,0.12,0.2,0.28)$

Then, the weighting-standardized matrix is:

$$
Z=\left[\begin{array}{lllll}
0.1037 & 0.0331 & 0.0465 & 0.0962 & 0.1878 \\
0.1383 & 0.1327 & 0.0558 & 0.0577 & 0.0876 \\
0.1244 & 0.0663 & 0.0651 & 0.1347 & 0.1461 \\
0.1106 & 0.0498 & 0.0698 & 0.0962 & 0.1044
\end{array}\right]
$$

\subsubsection{Getting the positive and negative ideal solutions}

$$
\begin{aligned}
& A^{+}=\left\{z_{1}^{+}, \cdots, z_{n}^{+}\right\}=\left\{\left(\max _{i} z_{i j} \mid j \in I\right),\left(\min _{i} z_{i j} \mid j \in J\right)\right\} \\
& A^{-}=\left\{z_{1}^{-}, \cdots, z_{n}^{-}\right\}=\left\{\left(\min _{i} z_{i j} \mid j \in I\right),\left(\max _{i} z_{i j} \mid j \in J\right)\right\}
\end{aligned}
$$

Note: $\mathrm{I}$ is the index set of the income attribute, $\mathrm{J}$ is the index set of cost attribute.

So, the positive-ideal solution and the negative-ideal solution can be obtained as follows:

$$
\begin{aligned}
& A^{+}=\{0.1383,0.1327,0.0698,0.1347,0.1878\} \\
& A^{-}=\{0.1037,0.0331,0.0465,0.0577,0.0876\}
\end{aligned}
$$

\subsubsection{Computing the distance of every concept to the ideal solution and negative ideal solution}

The distance to the positive-ideal solution $A^{+}$is:

$$
d_{i}^{+}=\sqrt{\sum_{j=1}^{n}\left(z_{i j}-z_{j}^{+}\right)^{2}}, i=1,2, \cdots, m
$$

The distance to the negative-ideal solution $A^{-}$is:

$$
d_{i}^{-}=\sqrt{\sum_{j=1}^{n}\left(z_{i j}-z_{j}^{-}\right)^{2}}, i=1,2, \cdots, m
$$

So, the distance of every scheme to the positive-ideal solution and negative-ideal solution can be obtained respectively as follows:

$$
\begin{array}{llll}
d_{1=0.1146}^{+} & d_{2=0.1271}^{+} & d_{3=0.0798}^{+} & d_{4=0.1268}^{+} \\
d_{1=0.1073}^{-} & d_{2=0.1058}^{-} & d_{3}^{-}=0.1060 & d_{4=0.0513}^{-}
\end{array}
$$

3.2.4 Calculating the relative similarity scale of every scheme

$$
R C_{i}=\frac{d_{i}^{-}}{d_{i}^{-}+d_{i}^{+}}, i=1,2, \cdots, m
$$

By introducing the connection vector distance, the relative similarity scale is calculated, which to some degree improves the traditional TOPSIS method. So, the relative similarity scale of every scheme is obtained as follows:

$$
\begin{array}{ll}
R C_{1=0.4836} & R C_{2}=0.4543 \\
R C_{3}=0.5705 & R C_{4}=0.2880
\end{array}
$$

\subsubsection{Ranking the pros and cons of each alternative}

Ranking the pros and cons of each alternative according to the numeric value of $\mathrm{RC}_{\mathrm{i}}$ in the descending order. The ranking result of the proposed scheme is acquired as follows:

$$
A_{3} \succ A_{1} \succ A_{2} \succ A_{4}
$$

Therefore, Car A3 (Maximum speed: $180 \mathrm{~km} / \mathrm{h}$, Price: $¥$ 200,000, Acceleration time: 14 s/100m, Reliability: 
High, Sensitivity: High) is the best choice.

\section{Multi-objective decisions making based on AHP}

\subsection{Basic principles of AHP}

AHP (Analytic Hierarchy Process) is one of the multiobjective decision methods combining qualitative and quantitative analysis. It is a modelling and quantificational process of complex decision-thinking systems of decision makers.

The basic idea of AHP is to decompose the complex decision problems into several hierarchies according to the general goal. Generally, the structure of multi-objective decision making problems can be divided into the following layers: the total purpose, the evaluation criteria and the specific measurements. Then, acquiring the relative importance of each factor through the multiple comparison at lower layer, and constructing the judgement matrix. After that, calculating the weight of each scheme for the total target by weighted-sum approach. The final conclusion is suggested that the maximum weight is the optimal program.

\subsection{Practical application of TOPSIS}

Decision maker will consider multiple factors and draw up various plans when purchasing cars. The decision maker $\mathrm{X} 2$ holds the view that the price, quality and external appearance are the three determining factors of final consumption after a preliminary analysis on the styles and accessories of several vehicles. Subsequently, the decision maker X2 formulates three purchasing options: an ordinary household vehicle, a high-end commercial vehicle, cheap second-hand vehicle. Table 3. summarizes the cars information collected by the decision maker X2 .

Table 3. Selection information list of 3 types of cars

\begin{tabular}{|c|c|c|c|}
\hline & Price & Quality & Appearance \\
\hline $\begin{array}{c}\text { Ordinary } \\
\text { household } \\
\text { vehicle }\end{array}$ & $¥ 120,000$ & $\begin{array}{c}4 \text { gears, } \\
\text { Automated } \\
\text { manual } \\
\text { transmission, } \\
7.3 \mathrm{~L} / 100 \mathrm{~km}\end{array}$ & $\begin{array}{c}\text { Silver grey, } \\
\text { Hatch-back } \\
\text { car }\end{array}$ \\
\hline $\begin{array}{c}\text { High-end } \\
\text { commercial } \\
\text { vehicle }\end{array}$ & $¥ 180,000$ & $\begin{array}{c}\text { Automated } \\
\text { manual } \\
\text { transmission, } \\
7.6 \mathrm{~L} / 100 \mathrm{~km}\end{array}$ & $\begin{array}{c}\text { Black, } \\
\text { Saloon car }\end{array}$ \\
\hline $\begin{array}{c}\text { Cheap } \\
\text { second-hand } \\
\text { vehicle }\end{array}$ & $¥ 80,000$ & $\begin{array}{c}\text { M gears, } \\
\text { Manual } \\
\text { transmission, } \\
6.3 \mathrm{~L} / 100 \mathrm{~km}\end{array}$ & $\begin{array}{c}\text { Red, Hatch- } \\
\text { back car }\end{array}$ \\
\hline
\end{tabular}

\subsubsection{Constructing hierarchy structure model}

The decision-making problem is generally divided into several levels -- the target layer, rule layer, program layer. The program to build a hierarchical structure model with different forms of block diagram indicating the hierarchical structure level and affiliation elements. Figure 1. demonstrated the hierarchy structure model in three layers.

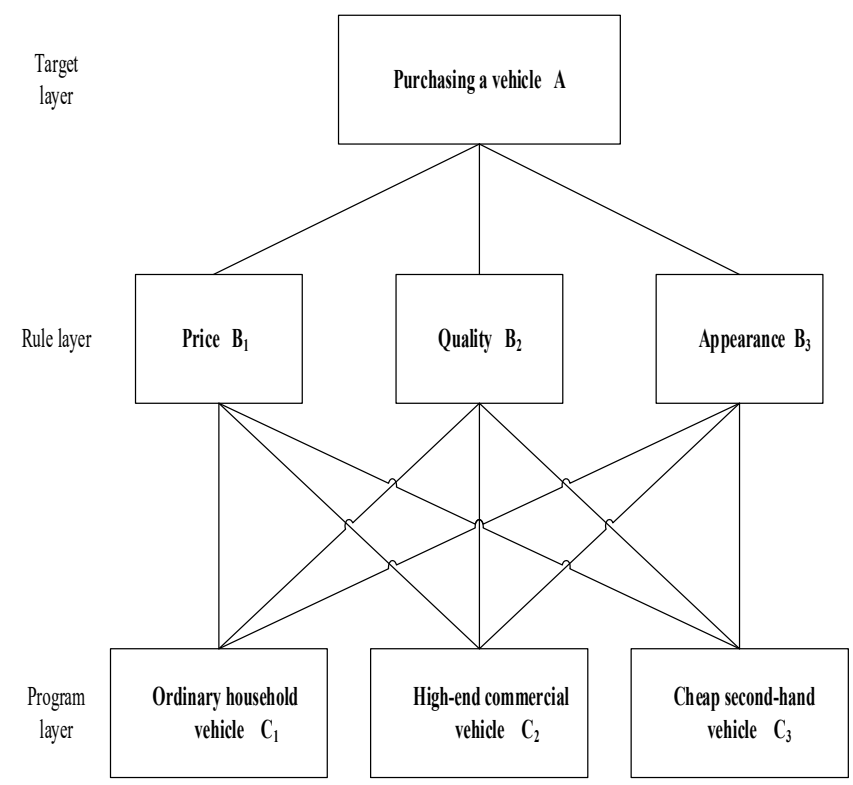

Figure 1. Hierarchy structure model

\subsubsection{Structuring matrixes}

The flexibility feature of AHP can reflect the unique preferences of each decision maker. Because its considered criteria can be varied with different individuals.

In order to measure the relative importance of each standard, AHP utilizes fractional score of 1 to 9 . In the pair-wise comparison matrix made up of 3 standards, Score 1, 3, 5, and 7 correspond to commonly important, fairly important, very important, and extremely important respectively.

For the general objective "purchasing a reasonable

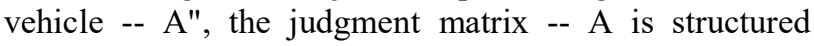
through multiple comparison among 3 rules, that is Low price -- B1, High quality -- B2 and Good exterior appearance -- B3.

The judgment matrix A can be obtained as follows:

$$
A=\begin{array}{c|ccc}
A_{1} & B_{1} & B_{2} & B_{3} \\
\hline B_{1} & 1 & \frac{1}{5} & 3 \\
B_{2} & 5 & 1 & 7 \\
B_{3} & \frac{1}{3} & \frac{1}{7} & 1
\end{array}
$$

Then, the matrix B is normalized from the elements of judgement matrix A by column.

$$
B=\left[\begin{array}{lll}
0.16 & 0.15 & 0.27 \\
0.79 & 0.74 & 0.64 \\
0.05 & 0.11 & 0.09
\end{array}\right]
$$




\subsubsection{Consistence validation}

(1) The weight is obtained from the matrix B:

$$
\mathrm{w}=(0.194,0.723,0.083)^{T}
$$

(2) Getting the Aw.

$$
\begin{gathered}
A \mathrm{w}=\mathrm{n}^{*} \mathrm{w}=\mathrm{n} *\left(\mathrm{w}_{1}, \mathrm{w}_{2}, \ldots \mathrm{w}_{\mathrm{n}}\right)^{\mathrm{T}} \\
A \mathrm{w}=(0.588,2.274,0.25)^{T}
\end{gathered}
$$

(3) Then, calculating the greatest eigenvalue $\lambda_{\max }$.

$$
\lambda_{\max } \approx \frac{1}{n} \sum_{i=1}^{n} \frac{(A w)_{i}}{w_{i}}=\frac{1}{n} \sum_{i=1}^{n} \frac{\sum_{j=1}^{n} a_{i j} w_{j}}{w_{i}}
$$

So, the maximum eigenvalue $\lambda_{\max }=3.062$

(4) Computing the C.I. (Consistency Index)

$$
\text { C.I. }=\frac{\lambda_{\max }-1}{n-1}
$$

So, the Consistency Index C.I. $=0.031$

(5) Computing the C.R.

Firstly, querying the R.I. (Random Index). As we known, R.I. is the consistency index of each pair-wise comparison matrix and its value depends on the number of items in the comparison. When $n=3$, R.I. $=0.58$; When $n=4$, R.I. $=0.90$.

So, R.I. $=0.58$

Secondly, calculating the C.R. (Consistency Ratio).

$$
\text { C.R. }=\frac{C . I .}{R \cdot I .}
$$

If C.R. $>0.10$, there is inconsistency between pair-wise comparisons. Otherwise, if C.R. $<0.10$, it shows that the consistency design of pair-wise comparisons is reasonable.

C.R. $=0.053<0.10$

So, the above-mentioned consistency design of pairwise comparisons is reasonable.

\subsubsection{The results analysis of AHP}

As mentioned above, the consistency result of the paired comparison matrix made up of 3 rules reaches the standard requirement. It can be concluded that the consistency of the pair-wise comparisons is acceptable and the matrix A has satisfactory consistency.

From the weight value -- w, we can see that the proportion of the second scheme is the highest. Hence, according to above-mentioned analytical result, it is more appropriate for the decision maker $\mathrm{X} 2$ to purchase the "High-end commercial vehicle" based on the judgment matrix.
The results analysis provides a basis for decisionmakers to better comprehend the balance of interests in the decision-making process, and also make it clearer to understand the reasons that the high-end business car is recommended by AHP method.

\section{Summary and discussion}

As assistant decision-making tools, TOPSIS and AHP have obvious advantages, such as practicality, conciseness and systematisms. As a result, the TOPSIS and AHP method can be introduced into solving the multi-objective decision-making problems. Based on the two methods, the proposed decision system can be applied for the quantitative sorting and rational selection of the alternative models, which is beneficial to the scientification and standardization of the decision-making.

However, the decision-making system should be amended according to the specific circumstances of the enterprises, due to the large number of criteria and rules involved in life.

When applying TOPSIS and AHP into multi-objective decision problems, we should pay highly attention to the following tips: The main factors supposed to be tightly grasped while decomposing and simplifying the multiobjective decision-making problems, not to speak of leaking or adding primary elements. What's more, the factors which are sufficiently different cannot be compared on the same layer.

\section{References}

1. W. Q. Liu, G.F. Yu, C. G. Xu, C\&D, Incentive Strategy Feasible Solution for Multi-objective Decision Making, 28, 957-960 (2015).

2. G. Y. Yang, Y. H. Zhang, MIPAT, Research on Solving Method of Multi-Objective Decision-making, 42, 108-115 (2012).

3. P. Y. Yuan, ERG, Decision Making of Individual Car Purchase Based on Analytic Hierarchy Process, 12, 67-75 (2015).

4. T. Cheng, MBTI, An Overview of the Combination of AHP and Multi-objective Methods, 25, 181-182 (2017).

5. S. K. PATIL, R. KANT, ESWA, A Fuzzy AHPTOPSIS Framework for Ranking the Solutions of Knowledge Management Adoption in Supply Chain to Overcome its Barriers, 41, 679-693 (2014).

6. D. M. ROIJERS, ICOAI, Efficient Methods for Multiobjective Decision-theoretic Planning, 24, 4389-4390 (2015).

7. I. A. BAKY, AMM, Interactive TOPSIS Algorithms for Solving Multi-level Non-linear Multi-objective Decision-making Problems, 38, 1417-1433 (2014).

8. B. B. SUN, X. Y. SU, CCN, Supplier selection decision method based on AHP/ Entropy /TOPSIS Algorithm, 7, 127-130 (2014). 